Julia Maria Kubica ${ }^{1}$, Krzysztof Pstrągowski ${ }^{1}$, Piotr Adamski², Michał Kasprzak¹, Ewa Obońska ${ }^{1}$, Karolina Obońska ${ }^{1}$, Anita Dąbrowska ${ }^{3}$, Salvatore Di Somma ${ }^{4}$, Andrzej Kleinrok ${ }^{5}$, Marcin Gruchała ${ }^{6}$, Piotr Kasztelowicz ${ }^{7}$, Przemysław Sobczak ${ }^{1}$, Przemysław Paciorek ${ }^{1}$, Marek Koziński², Jacek Kubica ${ }^{1}$

${ }^{1}$ Department of Cardiology and Internal Medicine, Collegium Medicum, Nicolaus Copernicus University, Bydgoszcz, Poland

2Department of Principles of Clinical Medicine, Collegium Medicum, Nicolaus Copernicus University, Bydgoszcz, Poland

${ }^{3}$ Department of Theoretical Foundations of Biomedical Science and Medical Informatics, Collegium Medicum,

Nicolaus Copernicus University, Bydgoszcz, Poland

${ }^{4}$ Emergency Department Sant'Andrea Hospital, Medical-Surgery Sciences and Translational Medicine, University La Sapienza, Rome, Italy

${ }^{5}$ Department of Cardiology, Pope John Paul II Hospital, Zamość, Poland

6I Department of Cardiology, Medical University of Gdańsk, Gdańsk, Poland

${ }^{7}$ Department of Internal Diseases, District Hospital in Chełmża, Poland

\title{
Mild therapeutic hypothermia for patients with acute coronary syndrome and cardiac arrest treated with percutaneous coronary intervention (UNICORN). The design and rationale for the prospective, observational, multicenter study
}

\author{
Corresponding author: \\ Piotr Adamski, MD, PhD \\ Department of Principles \\ of Clinical Medicine, \\ Collegium Medicum, \\ Nicolaus Copernicus University, \\ Skłodowskiej-Curie St. 9 , \\ 85-094 Bydgoszcz. Poland \\ Phone: +48525854023 \\ Fax: +48 525854024 \\ E-mail: piotr.adamski@wp.eu
}

Medical Research Journal 2016; Volume 1, Number 1, 23-27 10.5603/MRJ.2016.0004 Copyright @ 2016 Via Medica ISSN 2451-2591

\begin{abstract}
Introduction. Cardiac arrest constitutes the most frequent reason for sudden death in developed countries. Out-of-hospital cardiac arrest (OHCA) survivors are at high risk of death or neurologic deficits. The existing data regarding effectiveness and safety of mild therapeutic hypothermia (MTH) for treatment of OHCA survivors are inconsistent and ambiguous. Moreover, a uniform protocol of treatment by means of MTH is lacking. Methods. The UNICORN study is a phase IV, prospective, international, multi-centre, observational study designed to assess the effectiveness of MTH in patients after OHCA with shockable rhythm presenting with acute coronary syndrome (ACS). The trial is expected to include up to 500 patients. Depending on the availability of MTH in each study centre, besides the routine treatment of ACS in OHCA survivors, patients will either undergo MTH according to a uniform protocol or will not undergo MTH (250 patients per group). The primary end-point of the study is all cause mortality at 180 days after enrolment. Secondary end-points include: neurological outcome at discharge, stent thrombosis at 30 days, bleeding according to the BARC criteria, infectious complications at 180 days, and rhythm and conduction disorders at 180 days. Ethics and dissemination. The study received approval from the Local Ethics Committee to conduct the study (Komisja Bioetyczna Uniwersytetu Mikołaja Kopernika w Toruniu przy Collegium Medicum im. Ludwika Rydygiera w Bydgoszczy; study approval reference number KB 615/2015). The study results will be disseminated through conference presentations and publications in peer-reviewed journals.

Trial registration. ClinicalTrials.gov identifier: NCT02611934 (18 November 2015).

Key words: acute coronary syndrome, out-of-hospital cardiac arrest, percutaneous coronary intervention, therapeutic hypothermia
\end{abstract}

Med Res J 2016; 1 (1): 23-27

\section{Introduction}

Cardiac arrest constitutes the most frequent reason for sudden death in developed countries. It is estimat- ed that each year between 275,000 and 235,000 to 325,000 new cases of out-of-hospital cardiac arrest (OHCA) occur in Europe and the United States respectively $[1,2]$. The rate of admission to hospital after a suc- 
cessful return of spontaneous circulation in adult $\mathrm{OHCA}$ patients is estimated to be approximately $15 \%$ [3].

Unconscious patients admitted to intensive care units after OHCA are at high risk of death or neurologic deficits. The existing data regarding effectiveness and safety of mild therapeutic hypothermia (MTH) for the treatment of OHCA survivors are inconsistent.

Two relatively small randomized trials involving patients who remained unconscious after resuscitation from cardiac arrest of presumed cardiac cause with an initial shockable rhythm, compared therapeutic hypothermia $\left(32^{\circ} \mathrm{C}\right.$ to $34^{\circ} \mathrm{C}$ for 12 to 24 hours) with standard treatment. These studies showed a significant improvement in neurologic function and survival rates in patients treated with therapeutic hypothermia $[4,5]$. According to the current European Society of Cardiology (ESC) guidelines for the management of ST-elevation myocardial infarction, MTH is indicated early after resuscitation of cardiac arrest patients who are comatose or in deep sedation (class of recommendation I, level of evidence B) [6].

Dumas et al. performed a cohort study aimed at assessing the influence of percutaneous coronary intervention $(\mathrm{PCl})$ and therapeutic hypothermia on a long-term prognosis in resuscitated patients following OHCA [7]. MTH was applied in 241 out of 941 (25.6\%) OHCA survivors comatose at hospital admission. The highest survival rate was observed in patients treated with hypothermia and concomitant $\mathrm{PCl}$, while the worst outcomes were observed in patients who did not obtain any of these therapeutic methods. The authors concluded with the suggestion that the effects of acute hospital interventions for post-resuscitation treatment extend beyond hospital survival and can positively influence prognosis following the arrest hospitalization [7]. As none of the investigated methods ( $\mathrm{PCl}$ or hypothermia) used separately was as effective as the combination of both, we assumed that concomitant use of $\mathrm{PCl}$ and $\mathrm{MTH}$ is probably the optimal therapeutic approach to resuscitated patients with acute coronary syndrome (ACS) after OHCA.

A cohort study by Casella et al. provided a similar message [8]. MTH was performed in 141 (51\%) out of 278 comatose patients after OHCA. The authors demonstrated that in comatose patients resuscitated after OHCA emergency coronary angiography with $\mathrm{PCl}$, if indicated, in combination with MTH is associated with good neurologic outcomes and improved survival rates as compared with non-invasively managed cases. However, a more detailed analysis using a propensity-matched comparison and a multivariable analysis has not confirmed such benefits. Thus, these findings question the independent effect of the mentioned interventions and raise the possibility that the association of coronary angiography or $\mathrm{PCl}$ with a positive outcome, observed by others, could depend more on the pre-selection of a lower risk population that receives optimal post-OHCA care than on the benefits of early revascularization [8].

Favourable effect of MTH on survival and neurological outcome was confirmed in meta-analysis pooling data from randomized and non-randomized studies and in a recently published observational study with the historical control group [9, 10]. However, the results of the TTM trial (The Targeted Temperature Management Trial), the biggest available randomized study, are contradictory. Comparison of two treatment regimens: $\mathrm{MTH}$ at a targeted temperature of $33^{\circ} \mathrm{C}$ versus fever prevention at a targeted temperature of $36^{\circ} \mathrm{C}$ in comatose patients after $\mathrm{OHCA}$ did not provide any evidence of the superiority of MTH [11].

We found the methodology of this trial questionable, as there were significant limitations related to protocol and study conduction [11]. The protocol limitations were related to the non-uniform methodology of cooling and heterogeneous population of the trial. The intravascular cooling was used only in $24 \%$ of patients, while the remaining patients were cooled with surface method. The study population comprised $40 \%$ of patients with myocardial infarction, while myocardial necrosis was not confirmed in the remaining subjects. Moreover, there were also serious limitations related to the trial conduction. The target temperature was not reached in a substantial number of patients in the MTH arm as showed by the mean temperature during hypothermia. Huge dispersion of temperature during hypothermia exceeding $3^{\circ} \mathrm{C}$ was observed. The duration of the induction phase of hypothermia was unacceptably long with a mean of 8 hours [11]. These important shortcomings of the trial could have negatively affected its results.

Our own experience showed a $26 \%$ mortality rate in patients after OHCA treated with MTH and 54\% in the control group ( $p=0.005$; NNT = 3.55) [12], while in the TTM study, death occurred in $48 \%$ and $47 \%$ (ns) of patients respectively [11]. In our previous study, favourable neurological outcome was observed in 49\% of MTH patients and in $26 \%$ of patients in the control group ( $p=0.032$; NNT $=4.24$ ) compared with $46 \%$ and $48 \%$ (ns) in the TTM trial respectively. In our former trial [12], the intravascular cooling method was used in all $69 \mathrm{MHT}$ patients with support of cold saline infusion $\left(4^{\circ} \mathrm{C}\right)$ and ice packs at the induction of MTH compared with intravascular cooling applied only in $24 \%$ of the TTM study population with no defined induction proto$\mathrm{col}$ (at the discretion of the sites participating in the trial) as reported by Nielsen et al. [11]. Target temperature of $33^{\circ} \mathrm{C}$ was reached 4 hours after the initiation of $\mathrm{MTH}$ in our study (the mean in the study population), while in the TTM study the stabilization of temperature (above $33^{\circ} \mathrm{C}$ ) was observed after 8 hours, and the lowest temperature (still above $33^{\circ} \mathrm{C}$ ) after 17 hours. In both studies, the absolute temperature reduction in MTH patients amounted to $3.5^{\circ} \mathrm{C}$ and less then $2^{\circ} \mathrm{C}$, and concomitant $\mathrm{PCl}$ during 
hypothermia induction was performed in $75 \%$ and $42 \%$ of MTH patients respectively [11, 12].

In our cohort the following features were determined as independent predictors of in-hospital survival with favourable neurological outcome: OHCA with shockable rhythm (OR 12.36, Cl 95\% 1.15-132.4, $\mathrm{p}=0.035$ ), treatment with MTH (OR 4.65, Cl 95\% 1.19-18.25, $\mathrm{p}=0.0250$ ), reanimation duration (OR $0.57, \mathrm{Cl} 95 \%$ $0.38-0.85, \mathrm{p}=0.005$ ) and age (OR 0.41, Cl 95\% 0.25$-0.69, p=0.0006$ ) [12]. Substantial differences between the results of the above mentioned studies strongly suggest that careful patient selection and proper conduction of MTH procedure may have a pivotal impact on the results of resuscitated patients after $\mathrm{OHCA}[11,12]$.

We hypothesized that careful selection of patients with shockable rhythm and proper conduction of MTH procedure including quick induction up to the target temperature may improve clinical outcomes in resuscitated patients with diagnosed or suspected ACS after OHCA treated with concomitant $\mathrm{PCl}$, if feasible. Therefore, we have proposed to conduct a prospective, observational, multicentre study Mild Therapeutic Hypothermia for Patients with Acute Coronary Syndrome and Cardiac Arrest Treated with Percutaneous Coronary Intervention (UNICORN) [13].

\section{Methods}

\section{Study objectives}

The UNICORN study is designed to determine whether MTH applied in patients with ACS and car- diac arrest treated with $\mathrm{PCl}$ is associated with better clinical outcomes as compared with standard therapy without MTH.

\section{Study design}

The UNICORN trial is a phase IV, prospective, international, multi-centre, observational study. The study population includes unconscious subjects after OHCA with shockable rhythm presenting with ACS. After admission to one of the study centres and confirmation of the initial diagnosis, patients will be screened for eligibility for the study. Two types of study centres will participate in the trial: centres with and centres without MTH available. All study participants enrolled in centres with MTH available will be treated with MTH in addition to standard therapy, according to the protocol described below, and all study patients enrolled in centres without MTH available will be treated without this procedure (Fig. 1). The expected enrolment is 500 patients ( 250 patients per group). Apart from medical interventions related to $\mathrm{MTH}$, all patients in both study groups will be treated according to the same strategy (including in-hospital treatment, rehabilitation and long-term maintenance therapy). The planned follow-up is 180 days.

\section{Study population}

All adult patients with the score of $\leq 8$ on the Glasgow Coma Scale on admission to the hospital after OHCA with diagnosed or presumed ACS and shockable initial rhythm will be screened with regard to inclusion and exclusion criteria of the trial.

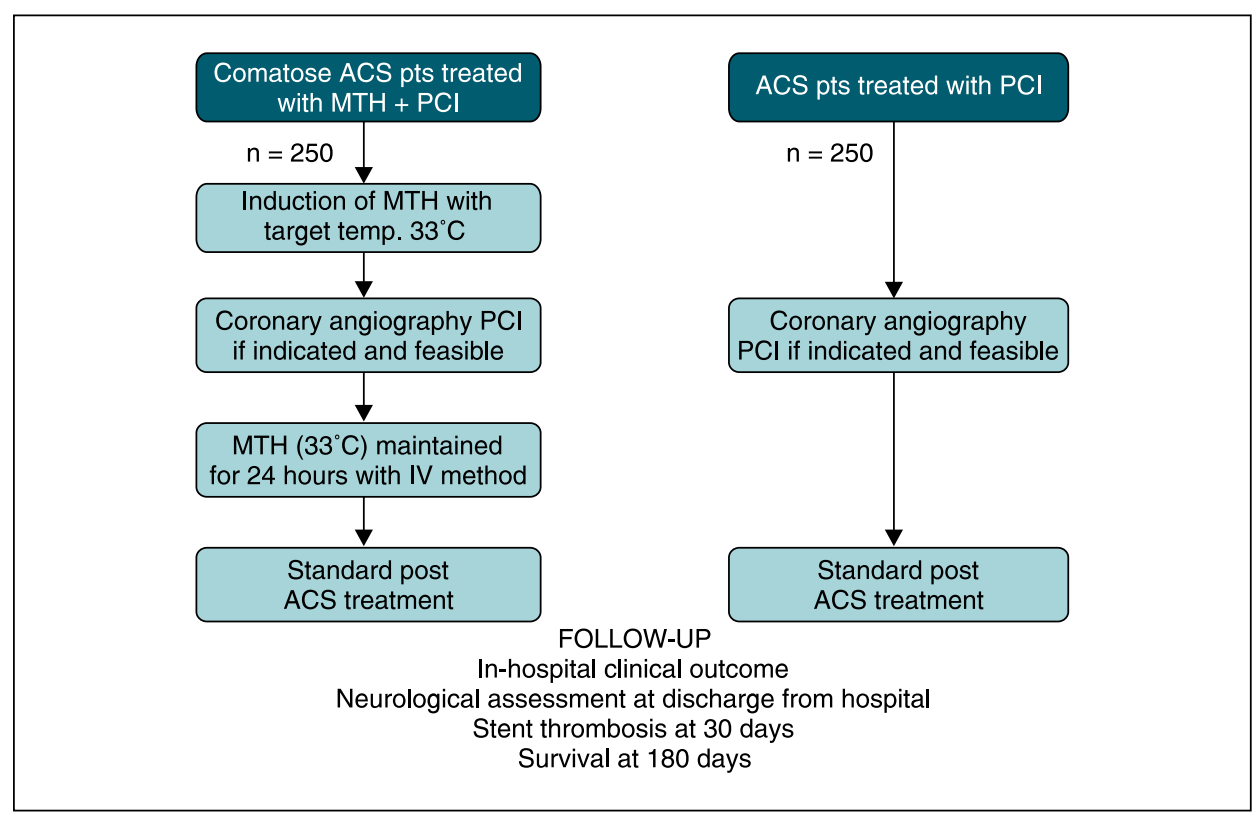

Figure 1. Flow chart of the UNICORN study; ACS - acute coronary syndromes; MTH - mild therapeutic hypothermia; $\mathrm{OHCA}$ - out-of-hospital cardiac arrest; $\mathrm{PCl}$ — percutaneous coronary intervention 


\section{Inclusion criteria}

The inclusion criteria are: 1 ) age $\geq 18$ years, 2) OHCA survivor, 3) sustained return of spontaneous circulation (ROSC) for more than 20 minutes after resuscitation, 4) unconsciousness with the score of $\leq 8$ on the Glasgow Co+ Scale after ROSC, 5) shockable initial rhythm, 6) diagnosis or suspicion of ACS.

\section{Exclusion criteria}

The exclusion criteria include: 1) unwitnessed OHCA, 2) obvious or suspected pregnancy, 3) known serious infection before OHCA, 4) known bleeding diathesis, 5) confirmed or suspected internal bleeding, 6) confirmed or suspected acute stroke, 7) confirmed or suspected cerebral injury, 8) known serious neurological dysfunction before OHCA (Cerebral Performance Category $[\mathrm{CPC}] \leq 4)$, 9) known serious disease making 180 days of survival unlikely, 10) hemodynamic instability with systolic blood pressure $<65 \mathrm{~mm} \mathrm{Hg}$ despite the treatment, 11) time delay from ROSC to MTH induction > 240 minutes, 12) asystole or pulseless electrical activity as the initial rhythm, 13) initial body temperature $<30^{\circ} \mathrm{C}$

\section{Treatment}

The induction of MTH will be performed with the infusion of a cold saline $(0.9 \%$ solution of sodium chloride at the temperature of $4^{\circ} \mathrm{C}$ ) and the usage of ice packs. MHT will be maintained with a MTH-dedicated catheter introduced into the inferior vena cava through the femoral vein by the interventional cardiologist during coronary angiography. MTH will be maintained for at least 12 hours, with an expected duration of 24 hours at the target temperature of $33^{\circ} \mathrm{C}$. The rewarming phase will be conducted in an actively controlled manner $\left(0.3^{\circ} \mathrm{C}\right.$ per hour $)$. The patient's core temperature will be independently measured in the urinary bladder as well as in the lower one third of the oesophagus using a dedicated catheter and tube.

All patients treated with MTH will be mechanically ventilated with a concomitant continuous intravenous infusion of propofol and fentanyl for sedation and analgesia.

During their participation in the study, all patients will be treated according to the current ESC guidelines. The choice of the access of the coronary invasive procedure (radial or femoral) and the type of implanted stent will be at the discretion of an operator. Moreover, standard therapy will include aspirin, P2Y12 receptor inhibitor, beta blockers, statins, and angiotensin-converting enzyme inhibitors or angiotensin II receptor blockers, if not contraindicated.

\section{Study end-points}

The primary end-point of the study is all cause mortality at 180 days after enrolment. Secondary end-points include: 1) neurological outcome defined according to CPC at discharge, 2) stent thrombosis at 30 days, 3 ) bleeding according to the BARC criteria, 4) infectious complications at 180 days, 5) rhythm and conduction disorders at 180 days. Patient risk stratification assessment will be performed according to plasma concentrations of natriuretic peptides (BNP or NTproBNP), ST2 and adrenomedullin at enrolment into the study [14-16].

\section{Statistical analysis}

The continuous variables in both study groups will be compared by means of the t-test for normally distributed values as assessed by Kolmogorov-Smirnov test. Otherwise, the Mann-Whitney $U$ test will be used. Proportions will be compared by the chi-square test when appropriate. Survival analysis will be performed by the Kaplan-Meier method with log-rank test group comparison.

\section{Determination of sample size}

We estimated that a sample of 454 patients would provide $90 \%$ power to detect a $30 \%$ reduction in the hazard ratio for death in the MTH group as compared with control group, at a two-sided alpha level of 0.05 . To detect a hazard ratio of death reduction of $30 \%$, with the assumption of mortality of $35 \%$ in the MTH group versus $50 \%$ in the control group, a sample of 227 patients per group would be needed. On the basis of these assumptions, a sample of 500 patients was chosen to allow for a drop-out of 46 patients.

\section{Ethics and dissemination}

\section{Ethics}

The study received a favourable ethical opinion and approval from the Local Ethics Committee to conduct the study (Komisja Bioetyczna Uniwersytetu Mikołaja Kopernika w Toruniu przy Collegium Medicum im. Ludwika Rydygiera w Bydgoszczy; study approval reference number KB 615/2015).

The study will be conducted in accordance with the principles contained in the Declaration of Helsinki. In accordance with national requirements and the principles of the Declaration of Helsinki, written informed consent will be waived, delayed, or obtained from a legal surrogate, depending on the circumstances, and will be obtained from each patient who regained mental capacity. 


\section{Present status}

The approval of the Local Ethics Committee was obtained on September 29, 2015. On November 18, 2015 the UNICORN study was registered on ClinicalTrials.gov (ClinicalTrials.gov identifier: NCT02611934)

The first patient is expected be enrolled in March 2016. We plan to complete the study enrolment in March 2018.

\section{Dissemination of results}

Treatment of all enrolled patients will be supervised online and consulted, if necessary, by UNICORN Clinical Committee. Data from all the study centres will be collected and analysed by central UNICORN Coordinating Laboratory.

The study results will be disseminated through conference presentations and peer-reviewed journals. The results will also be available to all participating clinicians to ensure the optimal care in OHCA patients.

\section{Summary}

The data on the efficacy of MHT in OHCA survivors is ambiguous and a uniform protocol for the procedure is lacking. The Unicorn study is expected to provide important information regarding the impact of $\mathrm{MTH}$ on clinical outcomes in OHCA survivors. Moreover, the study will assess whether stratification of patients according to concentrations of natriuretic peptides (BNP or NTproBNP), ST2, and adrenomedullin provides additional information regarding prediction of clinical outcomes in OHCA patients.

\section{Contributors}

JMK, KP and JK conceived the study. JMK, KP, PA and $\mathrm{JK}$ and wrote the study protocol with consultation from SDS, AK, MG, PK, PP and MK. Subsequently, JMK, KP, PA, MK, EO, KO, AD, SDM, AK, MG, PK, PP, MK and $\mathrm{JK}$ revised the manuscript critically for important intellectual content. All the authors read and approved the final manuscript.

\section{Funding}

The study is funded by Collegium Medicum of Nicolaus Copernicus University.

\section{Competing interests}

None declared.

\section{Ethics approval}

Komisja Bioetyczna Uniwersytetu Mikołaja Kopernika w Toruniu przy Collegium Medicum im. Ludwika Rydygiera w Bydgoszczy (reference: KB 615/2015).

\section{References}

1. Atwood C, Eisenberg MS, Herlitz $\mathrm{J}$ et al. Incidence of EMS-treated out- of-hospital cardiac arrest in Europe. Resuscitation 2005; 67: 75-80.

2. Nichol G, Thomas E, Callaway CW et al. Regional variation in out-of-hospital cardiac arrest incidence and outcome. JAMA 2008; 300: 1423-1431.

3. Stiell IG, Wells GA, Field B et al. Advanced cardiac life support in out-of-hospital cardiac arrest. N Engl J Med 2004; 351: 647-656.

4. Bernard SA, Gray TW, Buist MD et al. Treatment of comatose survivors of out-of- hospital cardiac arrest with induced hypothermia. N Engl $J$ Med 2002; 346: 557-563.

5. The Hypothermia after Cardiac Arrest Study Group. Mild therapeutic hypothermia to improve the neurologic outcome after cardiac arrest. N Engl J Med 2002; 346: 549-556.

6. Steg PG, James SK, Atar D et al. ESC guidelines for the management of acute myocardial infarction in patients presenting with ST-segment elevation: the task force on the management of ST-segment elevation acute myocardial infarction of the European society of cardiology (ESC). Eur Heart J 2012; 33: 2569-2619.

7. Dumas F. White L, Stubbs BA et al. Long-term prognosis following resuscitation from out of hospital cardiac arrest: role of percutaneous coronary intervention and therapeutic hypothermia. J Am Coll Cardiol 2012; 60: 21-27

8. Casella G, Carinci V, Cavallo P et al. Combining therapeutic hypothermia and emergent coronary angiography in out-of-hospital cardiac arrest survivors: Optimal post-arrest care for the best patient. Eur Heart J Acute Cardiovasc Care 2015; 4: 579-588.

9. Young-Min K, Hyeon-Woo Y, Seung-Hee J et al. Does therapeutic hypothermia benefit adult cardiac arrest patients presenting with non-shockable initial rhythms? A systematic review and meta-analysis of randomized and non-randomized studies. Resuscitation 2011; 83: 188-196.

10. Kozinski M, Pstragowski K, Kubica JM et al. ACS network-based implementation of therapeutic hypothermia for the treatment of comatose out-of-hospital cardiac arrest survivors improves clinical outcomes: the first European experience. Scand J Trauma Resusc Emerg Med 2013; 21: 547-556.

11. Nielsen N, Wetterslev J, Cronberg T et al. Targeted temperature management at $33^{\circ} \mathrm{C}$ versus $36^{\circ} \mathrm{C}$ after cardiac arrest. N Engl J Med 2013; 369: 2197-2206

12. Kubica JM, Pstragowski K, Koziński M et al. Mild therapeutic hypothermia for patients with acute coronary syndrome and cardiac arrest treated with percutaneous coronary intervention. A single center study [abstract]. Clin Chem Lab Med 2014; 52: eA467-eA468.

13. Kubica JM. Mild therapeutic hypothermia for patients with acute coronary syndrome and cardiac arrest treated with percutaneous coronary intervention (UNICORN). Research proposal [abstract]. Clin Chem Lab Med 2014; 52: eA467.

14. Lassus J, Gayat E, Mueller $\mathrm{C}$ et al. Incremental value of biomarkers to clinical variables for mortality prediction in acutely decompensated heart failure: the Multinational Observational Cohort on Acute Heart Failure (MOCA) study. Int J Cardiol 2013; 168: 2186-2194.

15. Farmakis D, Filippatos $\mathrm{G}$, Tubaro $\mathrm{M}$ et al. Natriuretic peptides in acute coronary syndromes: prognostic value and clinical implications. Congest Heart Fail 2008; 14 (suppl. 1): 25-29

16. Richards AM, Di Somma S, Mueller T. ST2 in stable and unstable ischemic heart diseases. Am J Cardiol 2015; 115 )suppl. 7): 48B-58B. 